\title{
CHRONIC RECURRENT MULTIFOCAL OSTEOMYELITIS: CLINICAL, LABORATORIAL AND TREATMENT FEATURES
}

Maraisa Centeville ${ }^{1}$, Roberto Marini ${ }^{1}$, Simone Appenzeller ${ }^{1, *}$

1.Universidade Estadual de Campinas, Campinas (SP), Brazil.

*Corresponding author: appenzellersimone@gmail.com

\section{BACKGROUND}

Chronic recurrent multifocal osteomyelitis (CRMO) is a rare inflammatory disease that presents with painful multifocal bone lesions. CRMO, an uncommon cause of bone pain, can occur isolated or as symptom of monogenic autoinflammatory condition. The insufficiency of awareness about the condition often postpone the diagnosis. Our aim was to describe the clinical, laboratory and treatment features of CRMO four cases followed in our outpatient's clinic.

\section{MATERIALS AND METHODS}

We did a retrospective study with charge review of CRMO cases followed at our outpatient's clinic. Clinical data, laboratory findings, treatment and outcomes were reported.

\section{RESULTS}

We followed four cases of CRMO, 2 males and 2 females, mean age of 9 years at diagnosis ( $\min 7$; max 14 ), mean time interval to diagnosis was 9.2 months ( $\min 2$; $\max 19$ ), differential diagnosis of infection and malignancy excluded in all cases, bone biopsy was compatible with chronic osteomyelitis in 3 cases and acute osteomyelitis in 1. At diagnosis computed tomography (CT), magnetic resonance imaging (MRI) or scintigraphy were performed in all cases, showing a range of 2 to 7 lesions per patient. Medium follow up time was 8.7 months ( $\min 1$; max 15). No treatment was required by 1 patient, 1 was treated with nonsteroidal anti-inflammatory drug (NSAID) and 2 used NSAID and pamidronate. All patients achieved remission of symptoms.

\section{CONCLUSION}

Chronic recurrent multifocal osteomyelitis is a rare disease which needs a high index of suspicion to get an early diagnosis. Our series treatment with NSAID and pamidronate seems to be effective in controlling the symptoms. 